\title{
Hablando del CurRículo InTEgrado DE JAMES BEANE*
}

\author{
Guillermo Contreras López
}

1.- Pertenecemos a una cultura en la que se da por sentado el hecho que la educación y el conocimiento se relacionan estrechamente, más aún cuando en general se entiende el concepto de educación fuertemente ligado al de escolarización. Sin embargo, el carácter de tal relación no es tan obvio ni goza de tan amplio consenso, ya que, a lo largo de la historia, ella ha demostrado estar dotada de un exlrardinario dinamismo. En primer lugar, el conocimiento no es un monumento inmutable. Por el contrario, en tanto contenido epistémico socialmente disponible, haly en el conocimiento una propensión al cambio en cuya virtud el prestigio adquirido por un concepto, contenido o teoría en un momento inicial se desgasta y desaparece tan pronto como el colectivo de pensamiento que lo sostiene le quita sus respaldo o es reemplazado por otro más potente. En consecuencia, lo que en un instante histórico es conocimiento al instante siguiente deviene en supercbería, creencia de gente antigua o cualquiera otra denominación o mote con que el lenguaje de un emergente grupo de poder designe a un conocimiento defenestrado. En segundo lugar, la educación, en su condición de operación política de acreditación de la generación de recambio, también va a estar dotada de dinamismo: tendrá definiciones, modalidades, finalidades cambiantes, para responder cada vez a las cambiantes relaciones de poder que se entabla al interior de la sociedad. Nada de extraño tiene, por tanto, que la relación entre dos dinamismos sea ella misma dinámica, cambiante, movediza. El tema es especialmente relevante en nuestro país en este momento, en el contexto de la reforma de la educación básica y media actualmente en curso. En el nivel medio la reforma apunta a corregir graves deficiencias, a saber, que lo que la educación actual propone a los jóvenes estudiar "no tiene significado ni valor para sus vidas" y "muestra carencias muy serias en términos de lograr competencias propias de una formación general de calidad." (Ministerio de Educación, 1997, Pág. 17).

2.- En la concepción academicista tradicional -anterior incluso al surgimiento cle las teorías del currículo- la meta de la educación es "reproducir el conocimiento acumulado con el propósito de conservar la herencia del pasado y de reconstruir los balsamentos que permitieron la conformación de una determinada área del

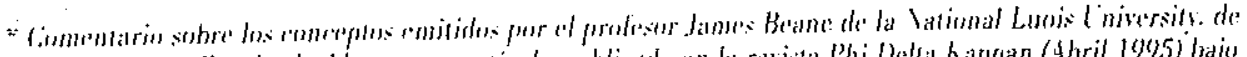

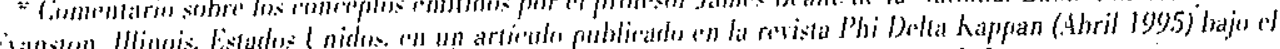
tilulo d $d^{2}$ Curriculum Integration and the Disciplines of Knowledge Tratulurevion de li. Ciontrerats
} 
saber" (Romeo,1998 pág. 59). Al efecto, se define el patrimonio cultural heredable como el conocimiento válido socialmente aceptado. Tenemos, pues, al conocimiento instalado en el centro mismo del programa educacional, definiendo el ser de la escuela, las actividades y relaciones de poder que surgen en su interior, la suerte de los estudiantes, el status de administradores y de profesores y toda una ideología y una moral que le dan sustento y protección. El vigor con que irrumpió en el escenario escolar la pedagogía por objetivos, durante el primer cuarto de este siglo, en la grupa del positivismo lógico, la psicología de la conducta, el eficientismo social, el management científico y el autoritarismo político, pareció en un principio cuestionar el prestigio del conocimiento como norte del proceso escolar. Así aparece en la postulación de Tyler:

"Ya que el auténtico propósito de la educación no consiste en que el instructor. realice determinadas actividades, sino en ocasionar cambios significativos en los patrones de comportamiento de los estudiantes,..." (Tyler; citado por Stenbouse, 1991, pág. 89).

No obstante, esa pedagogía no hizo sino afianzar tal prestigio. En lo concerniente al logro de objetivos cognitivos, la nueva pedagogía significó perseguir lo mismo que la anterior, pero con los resguardos y garantías técnicas suficientes para asegurar su éxito. Obsérvese que tanto para ser transmitido (y recepcionado) cuanto para ser recitado, repetido, analizado, sintetizado, aplicado, apreciado, etc. el conocimiento juega un rol absolutamente protagónico en los enfoques pedagó152 gicos mencionados.

3.- Entre las muchas propuestas de cambio curricular que hoy se pone a la consideración de las personas interesadas en el tema educacional destaca aquella del currículo integrado, o de integración del curriculo como se la denomina en el mundo anglosajón. Sin ser nueva, se trata de una propuesta original, por cuanto desplaza al conocimiento desde el centro del proceso educativo en que lo ubican los enfoques academicista y por objetivos y pone en ese lugar a lo sujetos del proceso, vale decir, a los estudiantes, sus carencias e inquietudes. De esta forma toma cuerpo una pedagogía para la cual lo más importante no es el acervo transmisible ni las estrategias conducentes a una transmisión eficiente y exitosa, sino un sujeto, cuyo menú de opciones presenta, entre otras, un reservorio epistémico. Esta propuesta es también radical en cuanto a su consideración de la cultura acumulada en el sentido que no solamente la saca del rol protagónico sino que le otorga otro subalterno, utilitario. Así, no se trata solamente de asignar al conocimiento un segundo o tercer lugar a título decorativo, como para volver la atención hacia él una vez que se haya completado etapas que le sean previas o de mayor urgencia. Por el contrario, se trata de ponerlo al servicio de procesos considerados de mayor jerarquía. Es decir, se lo convoca en todo instante del proceso educativo para finalidades que son superiores a él. Interesa al Profesor Beane dejar en claro el rol que cabe al conocimiento en un diseño curricular como el propuesto: "intencional y contextualmente ponemos al conocimiento a trabajar". Curiosamente, por el hecho de usar al conocimiento como herramienta para fines que lo trascienden se opera su des-reificación o, lo que es lo mismo, la re-vivificación del conocimiento. 
4.- No sigamos adelante sin antes poner de manifiesto que, al hablar de conocimiento, el autor se está refiriendo al conocimiento científico, corpus acumulado de contenidos epistémicos consistente en leyes, principios, propuestas teóricas, métodos de acción, modelos de pensamiento, etc., en constante crecimiento, refutación (al decir de Popper) y reformulación que forman nuestra explicación del mundo que nos rodea y que es producida por esa indagatoria humana sistemática y organizada que llamamos ciencia. Éste es el conocimiento que la escuela, según la definición de los enfoques pedagógicos tradicionales, ha recibido la misión de transmitir, el contenido y el continente de la buena vida. No sólo eso; es además el tipo de conocimiento desde el cual emanan los objetivos educacionales, según la definición de Ralph Tyler. En efecto, al analizar el proceso de selección de tales objetivos, este clásico del campo del currículo distingue tres fuentes que los originan, a saber, las necesidades e intereses de los estudiantes, la vida contemporánea fuera de la escuela y las sugerencias de "los especialistas en las distintas áreaś del conocimiento" (Tyler, en M. Leyton, 1969, pág. 91). Interesa aquí dejar en claro que cuando Tyler habla de las necesidades e intereses de los estudiantes se está refiriendo no a la percepción personal, subjetiva que de ellas tenga el profesor de aula sino a la definición que de ellas se haga luego de su auscultación, diagnóstico y medición por medio de los instrumentos proporcionados por la psicología y la sociología, disciplinas científicas que, en su época, hacían uso del mismo estilo indagacional que a Tyler le merecían confiabilidad. Vale decir, dato y destinatario eran definidos con arreglo al mismo paradigma.

Aquí se produce el primer desencuentro entre las pedagogías tradicionales y la propuesta del currículo integrado. En efecto, frente a aquéllas, que hacen emanar los objetivos educacionales del dato científico y del estado del conocimiento científico, nos indica el profesor Beane en el artículo en comento, que las fuentes del currículo deberían ser los problemas, tópicos y preocupaciones propuestas por la vida misma, las que provienen de dos esferas: 1) las preocupaciones del Yo o personales $y, 2$ ) temas y problemas propuestos por el mundo más amplio. Más aún, si el objeto último para el enfoque academicista es la transmisión y reproducción de los contenidos de la cultura acumulada y para la pedagogía por objetivos, "que el individuo cambie ciertas formas de conducta" (Tyler, en M. Leyton, 1969, pág. 71), para el currículo integrado el foco central es la búsqueda de significado personal y social. Es decir, para los enfoques tradicionales, la finalidad última consiste en transformar a la generación de recambio en heredera del patrimonio cultural de la humanidad precedente, definido éste en términos del conocimiento tenido por válido y de las pautas de relación que le dan sustento y legitimidad. Para el currículo integrado, el propósito es contribuir al encuentro de cada estudiante consigo mismo y con su entorno social.

Obviamente; el otro punto de desencuentro consistirá en los resultados que se espera del proceso educacional. Para los enfoques tradicionales, lo esperable será que el estudiante adquiera el status de reproductor de la cultura que heredó, sus contenidos y sus pautas de reproducción. Para el currículo integrado, por su parte, el logro es que los jóvenes incorporen "sus experiencias de aprendizaje en sus esquemas de significado para ampliar y profundizar su comprensión de sí mismos y del mundo", comenta Beane, que más adelante agrega que gracias a ello "empie- 
zan a buscar, adquirir y usar el conocimiento de manera orgánica, no artificial." fisto último equivale a decir que el contenido epistémico es invocado, en todo momento del proceso, para la solución de problemas definidos desde la percepción del propio estudiante. Resulta clara la filiación de esta propuesta con la teoría de los aprendizajes significativos de Ausubel (Ontoría, 1996 pág. 213) y las propuestas de Rogers en su Educación Centrada en el Alumno.

5.- Si bien lo expuesto hasta aquí da cuenta de una diferencia fundamental entre los enfoques pedagógicos tradicionales y el currículo integrado, no salta a la vistá ninguna distancia entre éste y las ciencias o disciplinas del conocimiento o conocimiento organizado. En opinión del profesor Beane la tensión surge a partir del esquema asignaturista en que se expresa en la organización de los planes de estudio tradicionales y de la postura que, en ese contexto, adoptan los profesores especialistas. Dice Beane (1995) que "el problema no es con las disciplinas del conocimiento en sí sino con su representación en un enfoque curricular de asignaturas separadas", porque "el tema no es si las disciplinas del conocimiento son útiles o no, sino cómo se las pòdría traer apropiadamente a las vidas de los jóvenes", más aún cuando no se sabe si ellas "incluyen todo lo que podría ser útil en la búsqueda de significado personal y social."

Desde luego, las disciplinas del conocimiento no son lo mismo que las asignaturas escolares. Aun cuando unas y otras comparten características, sus diferencias son del todo sustantivas y definitorias. Ciertamente, ambas compartimentan el saposible. De igual manera, asignatura y ciencia representada están abocadas a similares cuerpos de conocimientos. No obstante el propósito de la ciencia, sus técnicas e instrumentos, las experiencias que ofrece a sus cultores y las fronteras y limitaciones que reconoce a sus empeños difieren por completo de las finalidades, técnicas, experiencias y limitaciones de la asignatura escolar. Evidentemente, y sin menospreciar el objeto último de la actividad científica, la asignatura escolar tiene como propósito irrenunciable el contribuir a la formación intelectual, social y ética del estudiante. Dificilmente un científico aceptaría echar sobre sus hombros una tarea de esta naturaleza y, sin mirarla en menos, asume la suya propia, que es ampliar los límites de lo conocido en un campo indagacional determinado. En resumen, ciencia y asignatura no son la misma cosa. El hecho de que, a nivel escolar lleven el mismo rótulo le parece al profesor Beane una apropiación de nombre.

No obstante, lo anterior no quita status al profesor de asignatura ni disminuye mérito a su trabajo. El argumento sólo pretende dejar establecido que centrar el quehacer escolar en la construcción personal y social del estudiante no constituye un rechazo a nutrirse del cultivo y organización del conocimiento.

6.- La crítica del asignaturismo, sin embargo, trasciende los aspectos enunciados hasta aquí. Surge, más bien, de la consideración de, a lo menos, cuatro factores que lo acompañan indisolublemente. En primer lugar, la asignatura no contiene ni todo ni lo más reciente que ha logrado llegar a saber la ciencia que ella dice representar. De hecho, el listado de contenidos de la asignatura es solamente, y no 
podría ser más que, una selección lo más actualizada posible de los logros epistémicos de un campo del saber hecho por especialistas de ese campo y/o por profesores de esa asignatura. Es admisible al curriculo lo que esas personas estiman que vale la pena conocer; lo que según, su criterio, no valga la pena poner en el horizonte obligattorio de los estudiantes, será excluído. Y lo será no sólo de la formulación oficial conocida como plan de estudios. Se le excluirá también del texto -o de los rextos- que los editores correspondientes elaboren. Ingresamos aquí a un tema muy sensible. En efecto, una queja que se escucha cada vez con mayor frecuencia en boca de los especialistas en currículo y de profesores de aula es que las decisiones más importantes en la materia son tomadas por burócratas e industriales disminuyendo progresivamente el poder de los profesionales del área respecto de lo que se debe hacer en la escuela.

En segundo lugar, se critica al asignaturismo el haber transformado al conocimiento en un fin más que en un medio para la educación, en el entendido que las escuelas han asumido la tarea de congregar a jóvenes y a adultos con el objeto que los primeros lleguen a manejar datos, hechos, principios aislados, seleccionados para ellos por personas que tienen la autoridad social para hacerlo. No parece llamar a nadie la atención, excepto a alumnos y profesores en instantes de lucidez, que la transmisión de tales contenidos aislados, descontextualizados de la vida, reificados y no sometidos a validación en el tamiz de la vivencia personal carece de significado y está, como es obvio, condenada al olvido. Es el motivo por el cual Piaget observa que "nada se sabe con precisión sobre lo que queda, por ejemplo, de las enseñanzas de geografía o historia en la cabeza de un campesino de 30 años o sobre lo que un abogado ha conservado de los conocimientos de química, física o incluso geometría adquiridos en las clases del instituto." (Piaget, 1991, pág. 12). Y Jurjo Torres, por su parte, en vista del fracaso escolar inherente al esquema en comento, afirma que "la historia de la educación... puede ser releída también en sus efectos como la historia de la institucionalización y de la legitimación del fracaso escolar de la clase trabajadora y de los grupos sociales desfavorecidos" (Jurjo Torres, 1996, pág. 33).

En tercer lugar, y no obstante su alegato de privilegiar la transmisión de los contenidos cognitivos de la cultura acumulada con vistas al logro de determinadas competencias, el enfoque de asignaturas separadas es una vía inapropiada aún para los propósitos que sus defensores le atribuyen, opina el profesor Beane, y agrega que los jóvenes tienden a ejecutar por lo menos con igual perfección, y a menudo mejor, pruebas tradicionales de medición de logro escolar cuando el currículo avanza en la dirección de la integración. Sin necesidad de recurrir al sentido común, la experiencia parece indicarnos que cuando aprendemos lo hacemos no porque nos interese aprender, sino porque nos interesa resolver un problema cuya solución nos hemos autoimpuesto libremente.

Por último el asignaturismo involucra la idea, cargada de etnocentrismo, de que la única versión posible de la buena vida es aquélla ligada al conocimiento producido en áreas estrechamente definidas por varones blancos y de clase media alta, desmereciendo las formas de vida y de conocimiento surgidas en otros grupos sociales y de instancias productoras de saber independientes del ámbito académi- 
co. Al respecto, el autor citá a Michalel Apple, para quien la pregunta "¿cuál es el conocimiento más válido?" se ha reformulado en términos de "ia quién pertenece el conocimiento más válido?" (M. Apple. 1987, pág 38) o, dicho en sintaxis más simple iquién ba construido el conocimiento que más vale la pena transmitir?. Es clecir, incluso si aceptáramos que la misión de la educación y de la escuela es transferir conocimientos descle la generación adulta a la generación de recambio, y que por la enormidad de su corpus deberíamos seleccionar lo más relevante y excluir lo que consideráramos de menor relevancia, todavía deberíamos explicar por qué estaríamos incluyendo el saber de un grupo social y excluyendo el de otros. No se debe soslayar el hecho que el conocimiento validado para su inclusión en el currículo escolar, según opina el profesor. Beane en el artículo comentado, "funciona en favor de los jóvenes privilegiados en cuya cultura se lo encuentra regularmente, al tiempo que trabaja con severidad en contra de aquéllos provenientes de hogares no privilegiados y de culturas no dominantes" (Beane, 1995, pág. 618). O que, como apunta Gimeno, "los currícula dominantes suelen pedir a todos los alumnos lo que sólo unos pocos pueden cumplir (Gimeno, 1995, pág. 73).

7.- Si son tantos y de tanto bulto los defectos de la organización curricular asignaturista, uno se sorprende de su longevidad y supervivencia. En opinión de Beane, ella se sustenta en cuatro poderosos factores, a saber:

a) Las Elites Educacionales.- si el conocimiento es poder, también lo es la 156 gestión de su acrecencia, validación y divulgación. Inserta a la educación como articuladora de esta última, debe desarrollar un órgano que le permita llevar a cabo su misión; de allí, la formación de grupos y comunidades que, organizadas alrededor de las asignaturas, tienen el poder social para divulgar el conocimiento validado, formar a los divulgadores, organizarlos y proveerlos del material que les sea necesario. Al decir de Beane, ellos se encuentran entre "académicos,... supervisores,...editores de pruebas y textos, asociaciones de asignaturas y otros cuyos titulos y cargos a menudo denotan áreas asignaturales determinadas" (Beane, 1995, pág. 618). Difícilmente se puede esperar que ellos renuncien a sus respectivas cuotas de poder por muy persuasivos que sean los argumentos en favor de ello.

b) Las Expectativas y Exigencias de las Familias y la Prensa.- Ambas instancias apoyan la causa asignaturista, dice Beane, por su resistencia a aceptar para sus hijos una educación distinta de lo que recuerdan que fue la suya propia: En nuestra realidad chilena es probable que estos factores influyan de distinta manera. En lo concerniente a las familias, la tradición es que éstas deleguen en la escuela el desarrollo del educando y que padres y apoderados no se sientan con gran poder para hacer sentir su peso a la hora de decidir el tipo de educación que deben recibir sus hijos. En los estratos sociales de mayor poder adquisitivo, incluso, el apoderado se limita a elegir el establecimiento y a cautelar que tanto el trato como el currículo se desenvuelvan dentro de las normas de moralidad y respeto a las personas establecidas por la tradición. Dentro de ese ancho parámetro, se estima que la escuela sabe lo que hace y debe hacerlo.. La única exigencia es que la educación, adquirida a veces a alto costo, se justifique en términos de asegurar al pupilo su ingreso a la universidad si es que sus intereses o las expectativas familia- 
res están orientadas en esá dirección. En los estratos de menor poder adquisitivo operan similares parámetros, con la diferencia que, dado el carácter de asistencia social que asume toda prestación originada en la gestión pública, entre ellas la educación, y a la actitud de reconocimiento que en tal virtud la cultura chilena se ha habituado a requerir del beneficiario, las exigencias de éste son menores a la hora de cautelar la calidad de la educación y de evaluar sus resultados.

c) La Identificación de los Profesores.- A menudo, los profesores y supervisores construyen sus identidades a partir del asignaturismo, nos dice el profesor Beane. Agreguemos que contribuyen a esta construcción la institucionalidad escolar y la comunidad toda. En efecto, el título profesional encasilla al profesor y lo adscribe a una disciplina, los administradores le reconocen competencia exclusiva y excluyente para emitir pronunciamientos dentro - pero sólo dentro - de ella; se le otorga reconocimientos y estímulos (becas y pasantías) a partir de su expedición docente en ella, sus pares le reconocen autoridad por su dominio de su materia y será, para sus alumnos y apoderados, el profesor de inglés, la profesora de castellano, la señorita de biología, el señor de bistoria y no es raro escuchar tales vocativos incluso fuera del ámbito escolar. En una actividad caracterizada por retornos pecuniarios tan exiguos, tales vocativos constituyen rótulos no despreciables. Proponer un currículo no centrado en la asignatura puede ser percibido por los profesores como un salto hacia la nada.

d) El Período de Restauración Conservadora que el autor ve invadiendo su país. El enfoque del currículo integrado se enraíza en el movimiento progresista de la educación, y ello basta para ganarle el rechazo de los críticos conservadores.

El profesor Beane menciona en último término, y sin reconocerle el status de factor pro-asignaturista, un elemento que ciertamente juega en contra de una renovación curricular concebida en los términos de la propuesta integradora, a saber, el hecho que "el enfoque centrado en la asignatura está... enraizado en las estructuras profundas y en el folklore de la escolarización." (Beane, 1995 pág. 619). Ciertamente, éste es uno de los factores de mayor potencia, pues su remoción implica un cambio de la tradición y ello constituye palabras mayores.

8.- El escrutinio cada vez más agudo y fino de los defectos del asignaturismo, particularmente de su corrosivo efecto sobre las vidas de alumnos y profesores y sobre la relación que entre ellos se entabla en el curso de la praxis escolar, permitirá formarse un cuadro progresivamente más completo respecto de lo que los defensores del currículo integrado rechazan. Lo importante, sin embargo, una vez asumida la crítica, es adentrarse en lo que proponen y en el cómo lograrlo.

Primeramente, es preciso recordar que esta propuesta involucra devolver al lugar de privilegio en el status del profesor su carácter profesional, relegando la importancia de sus competencias técnicas a lugares secundarios. Al respecto es pertinente citar a Shirley Grundy:

"Cuando el trabajo de los profesores está informado por un interés técnico, aparece como manifestación de artesanía (o incluso de trabajo mecánico). Cuando 
las practicals que favorecen el aprendizaje se desilrollan de forma que dependen dé ejercicio del juicio príctico del autor, puede considerarse calracterizado por la protesionaliclad. El resultado de la protesionalidad es la acción príctica" (Grundy, 1994, pág. 2.13).

En segundo lugati, y parál no contraria los fundamentos doctrinarios del currículo integrado, deberemos abstenernos de pedir a sus defensores un recetario que nos indique qué hácer. La respuesta a tal inquietud, por el hecho de partir de la identificación cle los temals que resulten motivantes paral los jóvenes, debe surgir del ejercicio de la deliberación (Grundy, 1994, pág. 245) la negociación y la toma de clecisiones, procesos en los que las variables en juego son provistas por todos los actores escolatres pero cuyo foco está exclusivamente en los estudiantes, sus necesidades e intereses por construir significados y encontrar sentidos.

En tercer lugar, hay que entender que la propuesta en comento es una forma de ejercer la imaginación creadora y negociadorá, la que aparentemente corresponde a un órgano atrofiado por una vida y una cultura escolar construídas a partir de prácticas autoritarias y que, una vez puesto a trabajar, debería aumentar progresivamente tanto su presteza cuanto su productividad. En consecuencia, no debería calusar desánimo el hecho, muy previsible, de que los primeros ensayos tengan un éxito relativo o discutible.

9.- La etapa preliminar del procedimiento operativo consta de tres pasos, a 158 saber, identificación de los temas generadores, planificación y diseño de actividades. Si bien el autor reserva el primero de éstos al profesor, admite que "en casos de mayor sofisticación (esos temas) emergen de la planificación conjunta realizada con los estudiantes" (Beane, 1995, pág. 619). Es decir, se debe considerar el trabajo activo de los jóvenes -y no sólo su participación como referente tácito - desde la etapa preliminar, incluso en la identificación de los temas generadores, aun cuando advierte que esto último es posible sólo en un contexto de una mayor sofisticación. Por otra parte, indica que "no hay un paso intermedio en que se intente identificar qué asignaturas podrían contribuir al desarrollar el tema." (Beane, 1995. pág. 619) Es decir, la asignatura y su saber serán convocados no a priori sino a medida que se avanza en el desarrollo del tema. Al respecto, comenta el autor que "el trabajo hecho en el contexto de un currículo integrado es de suyo un currículo. No hay en la sala de espera otro 'currículo' esperando su turno de ser enseñado" (Beane, 1995, pág. 620). O, como ya sabíamos, lo que se hace al andar es propiamente el camino.

Dentro del marco conceptual esbozado en estos últimos párrafos, la siguiente cita es un ejemplo ofrecido al análisis de cómo los principios teóricos toman forma práctica.

"En una unidad sobre el 'ambiente' (los jóvenes) podrían crear simulaciones de diferentes biomas con artefactos reales o construídos y ofrecer tours guiados para mostrar sus trabajos, o podrían hacer experimentos respecto de los efectos de los contaminantes sobre el crecimiento de las plantas. O podrían montar y administrar un programa de reciclaje en la sala o en la escuela. O podrían identificar las materias primas de una serie de artículos de vestir e investigar su procedencia, $\therefore$ 
averiguar quién las fabrica y analizar los impactos ambientales y económicos del proceso completo. O podrían identificar problemas ambientales de su comunidad local y buscalr maneras de resolverlos." (Beane, 1995, págs.. 619-620)

Lia creatividad de cacla profesor o grupo-curso sumada al grado de sofisticación a que halyan llegado su identificación e inter-relación podrían prolongar ad infinitum la lista cle actividacles posibles. La idea es que cada actividad da origen a un proyecto: cada proyecto requiere de identificación, planificación, ejecución y evaLuación: cadal uná de estas etapas debe consultar el trabajo activo de los estudiantes, por lo cual ellas se constituirán de por sí en instancias de educación, socialización e instrucción; y, por último, el llevar cada una de ellas a cabo involucra la familiarización con -y el uso de - un determinado conocimiento susceptible de ser aportado por cualquier profesor actuando, en una primera aproximación, en su doble calidad de adulto informado o profesor generalista en temas integrados. Sólo en segundo lugar y a condición de que se requiera del auxilio de un conocimiento de mayor exclusividad se convocará al profesor como especialista en contenidos El conocimiento así convocado "se hace vivo, tiene significado y mayor posibiliclad de ser 'aprendido'".(Beane, 1995, pág. 620)

10.- Si clesde la óptica asignaturista se juzgara los logros cognitivos del currículo integrado, el autor advierte dos problemas. El primero, que es casi seguro que para la realización de un proyecto cualquiera los jóvenes requieran datos o informaciones más elaboradas que aquéllas que, en una secuencia hecha según un enfoque asignaturista corresponda a su nivel etario o estadio de desarrollo intelectual o nivel escolar. Es preciso tener en cuenta, al respecto, que una secuencia de ese tipo responde a una lógica, de las muchas posible. Se nos advierte al respecto que tales "secuencias varían de una escuela a otra y de un estado a otro,... son más arbitrarias de lo que desearían que creyéramos aquéllos que las construyen y defienden".(Beane, 1995, pág. 620) Probablemente, en un currículo integrado los procesos cognitivos se catapultan con una lógica distinta a la supuesta por el asignaturismo, o bien, la psicología del aprendizaje en que éste se fundamenta sea menos potente que aquélla que sustenta al currículo integrado, o bien, puede ser que cuando el interés es el acopio o despliegue retórico del conocimiento se convoca a ciertos inclusores (Ausubel, citado por Ontoría, pág. 22) -o se los convocal en un cierto orden - y cuando el interés es usarlo para la resolución de problemas, qiue pueden ser problemas del ser o del hacer, se convoque a otros inclusores o en un orden distinto.

Además de ellos, puede ocurrir que en el proceso de construcción de significados y búsqueda de sentido, los jóvenes no convoquen toda la información y babilidades diseminadas por la enseñanza asignaturista. A este propósito, el autor nos advierte que mucha de aquella información puede constituir banalidades o contenidos que sólo interesen a especialistas "e, incluso en tal caso, algo de tal información sería superflua." o verdades de ayer destinadas a disolverse en la alta marea del conocimiento de hoy. (Beane, 1995, pág. 620)

Si el interés primario de la integración del currículo es ampliar y profundizar nuestra comprensión de nosotros mismos y de nuestro mundo por medio de un aprendizaje, entendido éste como la integración continua de nuevos conocimientos y experiencias, se despriorizan completamente asuntos tan caros como la inte- 
gridad de la disciplina, la precisión de los límites entre una disciplina del saber y otra, la incuestionabilidad del horario tipo mosaico y el prestigio de una asignatura sobre otras (cosia propia e inseparable de la organización curricular asignaturista) y la sacralidad de la cultura dominante convertida en conocimiento. Nada de ello involucra, no obstante, un intento de concebir ni la escuela, ni la educación ni la vida sin el auxilio de la ciencia y sus logros. Por el contratio, concluye el autor, "las disciplinas del conocimiento no son lás enemigas del curriculo integrado" (Beane, 1995, pág. 622)

Frente a la tentación, asumida en ocasiones como un gravoso deber, de elaborar planes y programas de estudio cada vez más sofisticados, completos, actualizados, pertinentes, etc., en el contexto de una lógical educacional en cuyo primer plano no figure la producción de mano de obra calificada sino la formación de ciudadanos capaces de significar (construir internamente un mundo), significarse (desplegar esa construcción como producción económica y social que sea cooperativa y original a la vez) y aprender, la propuesta del currículo integrado es una opción nada despreciable.

\section{Bibliografía}

Apple, Michael Ideology and Curriculam, Routledge and Kegan Paul, Londres, $2^{3}$ ed. 1990 .

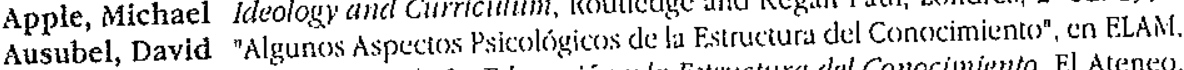
Stanley (comp), La Edacación y. la Estractura del Conocimiento, El Ateneo. Buenos Aircs., 1973, púgs. 211-238

Gimeno, José El Cumcio orictica del Curriculum. Morata, Nadrid, 2 ed., 1944.

Leyton, Mario Planeamiento Edacacional. Un wodelo Pudagógico, Edizorial Universitaria. Santiago, $3^{3}$ ed 1969 ,

Ministerio de Educación, Chile Objetivos Fundamentales y' Contenidos Minimos Obligatorios de la Educación Media, Versión para Consulta Nacional, Santiago, 1997.

Ontoria, A. Wapas Conceptuales. Una Técnica para Aprender, Editorial Narcea, Madrid, 6 ed 1996.

Piaget Jean Psicologia y Pedagogia. Ariel, Buenos Aires, 3 reimpresion, 1991.

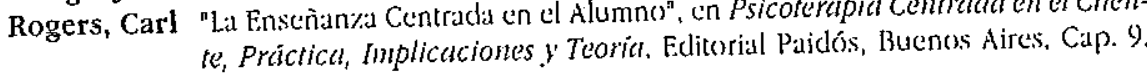
1975 págs 329-364,

Romeo, Julia Perspectivas del Cumiculam en la Educación Superior, en REVISTA ENFOQUES EDUCACIONALES, Departamento de Educación. Facultad de Ciencias Sociales, Universidad de Chile, 1998, (pp.57-74).

Stenhouse, Lawrence Investigación y' Desarrollo del Curricalo, Norata, Madrid, $3^{3}$ ed. 1991 Torres, Jurjo El curriculum Oculto, Morata, Madrid, ; ed. 1996

\section{$\Delta \rightarrow$ \\ Guillermo Contreras López

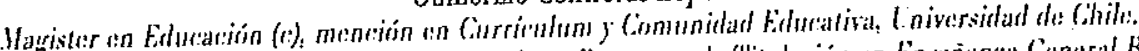

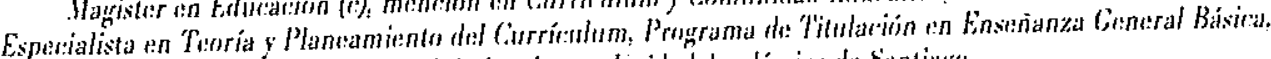 Conersidad de los Lages: I nitlad Iradímina de Santiagers.}

